\title{
New records and distributional data on the subtribe Staphylinina (Coleoptera: Staphylinidae) from Portugal
}

\author{
Raul Nascimento FERREIRA \\ 6 Fairview Dr.,Pawcatuck, CT 06379-1223 USA; email: insectcatcher@comcast.net
}

\begin{abstract}
Newly found species and new distributional records of the subtribe Staphylinina in Portugal are reported along with some comments on morphological characteristics of each genus. The current review of the subtribe includes 28 species in 8 genera, with two species new for the Portuguese fauna and 25 species and a subspecies recorded from new locations, expanding their geographic distribution.
\end{abstract}

Key words: Staphylinina, review, genus, species, distribution, fauna of Portugal

\section{INTRODUCTION}

The subtribe Staphylinina Latreille, 1802, according to the new reclassification concept (Smetana \& Davis 2000) have a ligula emarginated or bilobed, although the emargination is more or less minute, with the exception of some species of Dinothenarus s.str. The subtribe is represented in the Iberian Peninsula by nine genera: Abemus Mulsant et Rey, 1876, Creophilus Leach, 1819, Dinothenarus Thompson, 1858, Emus Leach, 1819, Ocypus Leach, 1819, Ontholestes Ganglbauer, 1895, Platydracus Thompson, 1858, Staphylinus Linnaeus, 1758 and Tasgius Stephens, 1829 with a total of 46 species and 5 subspecies (Gamarra \& Outerelo 2008). All of the nine genera are reported from Spain, but only eight from Portugal because the genus Abemus Mulsant et Rey, 1876 has not yet been found in Portugal.

The genera reported from Portugal are analyzed here with new species from Portugal as well as new localities, increasing their known distribution.

\section{MATERIAL AND METHODS}

The material for this study includes mainly specimens from the author's personal collection (RNFC), and from Father Ramiro Neves collection (RNC). The material was accumulated over a decade or more on trips to Portugal. During the trips, collecting was done by hand, pitfall and bait traps. A total of 156 specimens representing 28 species and 8 genera were studied.

New data includes: country, province, district, town, village or place.

\section{RESULTS}

Family Staphylinidae Latreille, 1802

Subfamily Staphilininae Latreille, 1802

Tribe Staphylinini Latreille, 1802

Subtribe Staphilinina Latreille, 1802

Genus Abemus Mulsant and Rey, 1876

Type species: Staphylinus chloropterus Panzer, 1796 
Species of this genus show mandibles similarly developed with tridentate middle teeth not on the same plane and the last segment of labial and maxillary palpi asetose and fusiform.

The genus is not yet known from Portugal, but if further research is done, it might be found in the southern part of Portugal in the Southeast areas of the Caldeirão and Figo Mountains. Abemus chloropterus (Panzer, 1796) of European distribution is known from Andalucia, Spain (Gamarra \& Outerelo 2008) with a Palearctic distribution.

Genus Creophilus Leach, 1819

Type species: Staphylinus maxillosus Linnaeus, 1758

The members of this genus are easily recognized and separated from other genera by the disc of the pronotum and dorsal face impunctate and shiny with the exception of the anterior angles which have a black grayish and yellowish dense pubescence. The body is covered with pubescence varying in color from black grayish, silver and golden to yellowish brownish red.

From Portugal only one species, Creophilus maxillosus (Linnaeus, 1758), is known to be found countrywide. Found during all the months of the year in carrion of all kinds, but not so frequently in decaying plant material or dung. The adults and larvae are predators of larvae and adults of Diptera and other arthropods attracted to the carrion. When disturbed they roll off the carrion onto the ground and if threatened they protrude their glandules emitting iridodial and other repelling secretions such as dihydroneptalactone, as a main ingredient of chemical defense.

Genus of Neotropical, Nearctic, Palearctic and Oriental distribution.

\section{Creophilus maxillosus (Linnaeus, 1758)}

Reported from Portugal for the first time by Fauvel as Emus maxillosus Linnaeus, 1758 without a locality. Paulino de Oliveira (1893), in his catalog, mentions it as Emus maxillosus Linnaeus, 1758 from the localities: Monchique, Beja, Coimbra and Braga. Later, Seabra (1943) mentioned it as Creophilus maxillosus Linnaeus, 1758 but as first cited by Fauvel and without any location. Ferreira (1962) recorded this species from Portugal. Gamarra \& Outerelo (2008) reported this species from the following Provinces: Minho, Beira Litoral, Estremadura, Ribatejo, Baixo Alentejo and Algarve.

New data. Portugal: Azores, Santa Maria, Vila do Porto 10 Jun 1966 (of. Dalberto Pombo), 1 ex. (RNFC); Beira Litoral, Coimbra, Coimbra (Choupal), 26 Jul 1966, 2 exx. (RNFC), 24 Jul 1968, 1 ex. (RNFC), Pinhal de Marrocos, 26 Jul 1966, 1 ex. (RNFC); Douro Litoral, Porto, Paços de Ferreira, Penamaior, 6 Nov 1969, 2 exx. (RNFC), Penamaior, no date, 1 ex. (RNC), without any reference, 4 exx. (RNC); Minho, Braga, Terras do Bouro, Serra do Gerês, 15 April 1972, 1 ex. (RNFC).

\section{Genus Dinothenarus Thomson, 1858}

Type species: Staphylinus pubescens DeGeer, 1774

Dinothenarus Thomson, 1858 is a Holarctic and Oriental genus with most of the known species found in the Palearctic region. Two subgenera are recognized: Dinothenarus sensu stricto with 9 species and Parabemus Reitter, 1909 with 19 species. Members of this genus have mandibular prostheca bilobed, with basal group of long cilia and right mandible with one broad tooth at middle and left mandible with two teeth or one stout one, and with maxillary palpus with apical segment elongate, distinctly fusiform, narrower and subequal in length to segment three (Smetana \& Davies 2000). 
Subgenus Dinothenarus Thomson, 1858

Dinothenarus (Dinothenarus) pubescens (De Geer, 1774)

Acording to Gammara and Outerelo (2008) this species had not yet been found in Portugal. It is mentioned in this paper for the first time.

New data. Portugal: Beira Litoral, Coimbra, Coimbra, Choupal, 16 May 1969, 1 ex. (RNFC).

Subgenus Parabemus Reitter, 1909

Type species: Dinothenarus fossor (Scopoli, 1771)

Dinothenarus (Parabemus) fossor (Scopoli, 1771)

Gammara \& Outerelo (2008) report this species from Algarve.

New data. Portugal: Beira Litoral, Coimbra, Coimbra, Vale de Canas, 16 June 1966, 2 exx. (RNFC); Algarve, Faro, Loulé, Vila Moura 10 August 1998, 1 ex. (RNFC).

Genus Emus Leach, 1819

Type species: Staphylinus hirtus Linnaeus, 1758

Species of this genus are very distinctive having the pronotum and portions of the abdomen with long, matted, black, golden yellow and whitish gray pubescence. Only one species exists in Portugal and its distribution is Palearctic.

\section{Emus hirtus (Linnaeus, 1758)}

Oliveira (1893) did not record this species for Portugal in his catalog. It was listed by Seabra $(1942,1943)$ from Serra do Gerês, attributing this species to Oliveira, as first mentioned from Portugal. Gamarra \& Outerelo (2008) mention this species from Beira Litoral without any locality.

New data. Portugal: Minho, Braga, Vieira do Minho, Serra do Gerês, Gerês 2 Aug 1969, 3 exx. (RNFC), 15 Apr 1972, 1 ex. (RNFC); 2 May 1973, 1 ex. (RNFC); Terras do Bouro, Serra do Gerês 15 April 1972, 1 ex. (RNFC); 2 May 1973, 2 ex. (RNFC).

Genus Ocypus Leach, 1819

Type species: Staphylinus ophthalmicus Scopoli, 1763

Ocypus Leach, 1819 is a Holarctic and Oriental genus with most known species found in the Palearctic and Oriental regions. Three subgenera are recognized for Portugal: Mantidus Motschulsky, 1860, Ocypus sensu stricto and Pseudocypus Mulsant et Rey, 1876. Members of this genus have the last segment of the labial palpus of variable fusiform shape, more or less parallel sided and apically truncate.

Subgenus Mantidus Motschulsky, 1860

Type species: Mantidus forficularius Motschoulsky, 1860

\section{Ocypus (Mantidus) nitens (Schrank, 1781)}

Oliveira (1893) mentioned this species from Portugal for the first time in his catalog as Staphylinus nitens Schrank, 1781 from Bussaco, Coimbra, Azambuja and Évora. Seabra (1943) referred to it as Staphilinus (Goerius) similis Fabricius, 1793 now a synonym of Ocypus nitens (Schrank, 1781) and nomen nudum. Ferreira (1962) listed this species from Portugal as 
Staphylinus similis nitens. Gamarra \& Outerelo (2008) mentioned the species from the following Provinces: Beira Litoral, Ribatejo, and Alto Alentejo. Holarctic distribution.

New data. Portugal: Beira Litoral, Leiria, Ansião, Avelar 1 Jul 1970, 1 ex. (RNFC).

\section{Subgenus Ocypus Leach, 1819}

\section{Ocypus (Ocypus) olens (Muller, 1764)}

Oliveira (1893) cited this species from Portugual and Seabra (1943) indicated it as first reported from Portugal by Fauvel. Ferreira (1962) mentioned this species from Portugal as Staphylinus olens. Gamarra \& Outerelo (2008) reports this species from Minho, Trás-osMontes e Alto Douro, Beira Litoral, Estremadura, Ribatejo, Baixo Alentejo and Algarve. Holarctic distribution.

New data. Portugal: Azores, Santa Maria, Vila do Porto, 16 Oct 1966, 2 exx. (of. Dalberto Pombo), 1 ex. (RNFC), without locality 3 exx. (RNC); Douro Litoral, Porto, Santo Tirso, Negrelos, no date , 2 exx. (RNC), Paços de Ferreira, Penamaior, no date, 3 exx. (RNC); Trás-os-Montes e Alto Douro, Bragança, Bragança, no date, 1 ex. (RNC); Beira Litoral, Coimbra, Coimbra, Choupal, 23 Jun 1961, 1 ex (RNFC), 27 Jun 1961, 1 ex. (RNFC), 8 Sep 1966, 1 ex. (RNFC), 4 Mar 1969, 1 ex. (RNFC), 24 Mar 1969, 1 ex. (RNFC), Olivais 20 Dec 1963, 1 ex. (RNFC), Penedo da Meditação 1 Apr 1961, 1 ex. (RNFC), Bairro Marchal Carmona, 24 Oct 1962, 1 ex. (RNFC), 10 Nov 1962, 1 ex. (RNFC), 12 Oct 1967, 1 ex. (RNFC), 24 Oct 1967, 1 ex. (RNFC), 10 Nov 1967, 1 ex. (RNFC), 11 Nov 1967, 1 ex. (RNFC), 2 Nov 1968, 1 ex. (RNFC), Calhabé, 2 Jun 1967, 1 ex. (RNFC), Pinhal de Marrocos, 6 Aug 1961, 1 ex. (RNFC), 23 Oct 1967, 1 ex. (RNFC), 5 Jun 1969, 2 exx. (RNFC), 7 Mar 1971, 1 ex. (RNFC), Celas, 12 May 1962, 1 ex. (RNFC), 20 May 1962, 1 ex. (RNFC), 3 Jun 1962, 1 ex. (RNFC), Arcos do Jardim 24 Nov 1967, 1 ex. (RNFC), Santo António dos Olivais, 15 Jun 1966, 1 ex. (RNFC), 8 May 1968, 1 ex. (RNFC), São José (Near Stadium), 24 Mar 1969, 1 ex. (RNFC), 7 Nov 1969, 1 ex. (RNFC); Leiria, Batalha, Regengo do Fetal, Serra d'Aire, 11 May 1950, 1 ex. (RNC), Ansião, Avelar, 2 Jun 1967, 1 ex. (RNFC); Estremadura, Lisboa, Lisboa, Cascais, São João do Estoril, 27 Oct 1968, 1 ex. (RNFC), Paredes, 4 Feb 1965, 1 ex. (RNFC), Sete Rios, 8 Feb 1966, 1 ex. (RNFC).

\section{Ocypus (Ocypus) ophthalmicus (Scopoli, 1763)}

This species is listed from Portugal by Oliveira (1893) from Monchique, Serra da Estrêla and Leça. Seabra (1943) stated that it was reported for the first time by Fauvel. Ferreira (1962) mentioned this species from Portugal as Staphylinus ophthalmicus. Gamarra \& Outerelo (2008) reported this species from Douro Litoral, Alto Douro, Estremadura and Algarve.

New data. Portugal: Minho, Viana do Castelo, Caminha, Foz do Rio Minho, Pinhal do Camarido, 24 Jun 1966, 1 ex. (RNFC); Trás-os-Montes e Alto Douro, Vila Real, Sabrosa, São Martinho de Antas, 4 Nov 1969, 2 ex. (RNFC), São Martinho de Antas, no date, 1 ex. (RNC); Beira Alta, Guarda, Manteigas, Torre (Estrada da Torre), 24 Jun 1970, 1 ex. (RNFC); Estremadura, Lisboa, Lisboa, Tapada da Ajuda (M.E.A.U.), 12 Nov 1968, 1 ex. (RNFC); Alto Alentejo, Évora, Alto dos Cucos, 4 Nov 1963, 1 ex. (RNFC).

\section{Ocypus (Ocypus) ophthalmicus ibericus Coiffait, 1964}

Coiffai (1964) described this subspecies from Cabo de São Vicente from a series of specimens of both sexes and stated 'C'est la forme signaleé sous le nom d' atrocyaneus de la Peninsule Ibérique le veritable atrocyaneus décrit de Mers-et- Kébir est special á l'Afrique du Nord." 
Gamarra \& Outerelo (2008) mention this subspecies as a synonym of atrocyaneus. More material is needed and the geographic distribution of both subspecies should be assessed with DNA-barcoding in order to define and test the subspecies boundaries.

I believe that only the subspecies atrocyaneus occurs in the South of Portugal, but further collecting must be conducted to establish the boundaries of both subspecies.

\section{Subgenus Pseudocypus Mulsant and Rey, 1876}

\section{Ocypus (Pseudocypus) aeneocephalus (DeGeer, 1774)}

Referenced by Oliveira (1893) from Beja, Guarda, Estarreja and Serra de Rebordaos.

Seabra (1943) indicated that it was mentioned by Heyden (1870) from Serra da Estrêla. Ferreira (1962) indicates this species from Portugal as Staphylinus aeneocephalus. Gamarra \& Outerelo (2008) mention this species from Trás-os-Montes e Alto Douro, Beira Alta, Beira Litoral, Estremadura and Baixo Alentejo.

New data. Portugal: Douro Litoral, Porto, Paços de Ferreira, Penamaior, no date, 1 ex. (RNC); Trás-os-Montes e Alto Douro, Bragança, Bragança, no date, 2 ex. (RNC)

\section{Ocypus (Pseudocypus) aethiops (Waltl, 1835)}

Oliveira (1893) mentioned this species “dans le sud du Douro je l'ai trouvé partout". De la Fuente (1922) cited this species from Lisboa by Quedenfeld (1884). Ferreira (1962) mentioned the species from Portugal as Staphylinus aethiops. Gamarra \& Outerelo (2008) mentioned this species from Douro Litoral, Beira Litoral, Estremadura and Algarve.

New data. Portugal: Minho, Viana do Castelo, Caminha, Foz do Rio Minho Pinhal do Camarido, 24 Apr 1966, 1 ex. (RNFC), 14 May 1966, 1 ex. (RNFC), 24 May 1966, 6 exx. (RNFC), 20 Jun 1966, 1 ex. (RNFC), 24 Jun 1966, 9 exx. (RNFC), Braga, Vieira do Minho, Serra do Gerês, 17 Jul 1966, 1 ex. (RNFC), Vila Nova de Famalicão, Famalicão, no date, 3 exx. (RNC); Douro Litoral, Porto, Paços de Ferreira, Penamaior, no date, 1 ex. (RNC); Trásos-Montes e Alto Douro, Vila Real, Chaves, Vidago, no date, 1 ex. (RNC); Beira Litoral, Aveiro, São João da Madeira, Quinta Neves 17 Jul 1966, 1 ex. (RNFC); Coimbra, Coimbra, Choupal 23 Jul 1961, 2 exx (RNFC), Celas, 20 May1967, 3 exx. (RNFC), Bairro Marchal Carmona, 12 Sep 1967, 1 exx. (RNFC), Ribeira dos Frades 3 Jun 1970, 1 ex. (RNFC); Leiria, Mata de Leiria, 1 Sep 1968, 1 exx. (RNFC), Ansião, Avelar, 6 Jul 1969, 3 exx. (RNFC), 16 Jul 1969, 2 exx. (RNFC); Estremadura, Lisboa, Lisboa, Vale do Jamor, 26 Jul 1965, 1 ex. (RNFC), Tapada da Ajuda (MEAU), 15 Nov 1968, 1 ex. (RNFC).

\section{Ocypus (Pseudocypus) fortunatarum Wollaston, 1871}

Gamarra \& Outerelo (2008) referred to this species from Estremadura and Alto Alentejo.

New data. Portugal: Beira Litoral, Leiria, Ansião, Serra de Sicó 2 Jul 1969, 1 ex. (RNFC).

\section{Ocypus (Pseudocypus) obscuroaeneus Fairmaire, 1852}

Gamarra \& Outerelo (2008) listed this species from the following Provinces: Beira Alta and Alto Alentejo without any localities.

\section{Ocypus (Pseudocypus) picipennis (Fabricius, 1793)}

This species was mentioned by Oliveira (1893) from Serra do Rebordao and Coimbra. Seabra ( 1943) indicated Oliveira as the first to report it from Portugal but is not included in his catalog. Quedenfeldt (1884) reports this species from Serra da Estrêla. Ferreira (1962) mentioned it from Portugal as Staphylinus picipennis. Gamarra \& Outerelo (2008) listed it from Trás -os-Montes e Alto Douro, Beira Alta, Beira Baixa, Beira Litoral and Baixo Alentejo. 
New data. Portugal: Minho, Viana do Castelo, Caminha, Moledo 26 Jun 1966, 1 ex. (RNFC); Beira Litoral, Coimbra, Coimbra, Mealhada, Bussaco, 12 Jun 1966, 1 ex. (RNFC); Estremadura, Lisboa, Lisboa, Tapada da Ajuda (MEAU), 22 Nov 1968, 1 ex. (RNFC); Alto Alentejo, Evora, Alto dos Cucos 4 Nov 1963, 3 exx. (RNFC).

\section{Ocypus (Pseudocypus) picipennis nevadensis (Muller, 1926)}

Gamarra \& Outerelo (2008) mentioned this species from Cataluña, Spain and from Portugal with a question mark. These reference must be verified for Portugal.

\section{Genus Ontholestes Ganglbauer, 1893}

Type species: Staphylinus murinus Linnaeus, 1758

The genus Ontholestes Ganglbauer, 1893 has anterior angles of the pronotum sharp and angulately of with 90 degrees or less, and mesosternum with fully developed long medial carina.

Genus of Neotropical, Nearctic, Palearctic, Ethiopian and Oriental distribution.

\section{Ontholestes marginalis (Gené, 1836)}

Ferreira (1962) listed this species from Portugal. Gamarra \& Outerelo (2008) mention this species with a question mark. Smetana (1959) states that it has been recorded from Portugal, Spain, Corsica, Sardinia and in North Africa from Algeria, Morocco and Tunisia.

\section{Ontholestes murinus (Linnaeus, 1758)}

Oliveira (1893) mentioned this species from Portugal as Leistrophus murinus Linnaeus, 1758 from Coimbra and Gerês. Seabra (1943) mentioned it from Portugal. Gamarra \& Outerelo (2008) recorded it from Minho, Trás-os-Montes e Alto Douro, Beira Litoral, Estremadura and Baixo Alentejo.

New data. Portugal: Minho, Braga, Terras do Bouro, Serra do Gerês, Gerês 6 Apr 1972, 1 ex. (RNFC); Douro Litoral, Porto, Paços de Ferreira, Penamaior, 10 Nov 1969, 1 ex. (RNFC), Penamaior, no date, 1 ex. (RNC); Trás-os-Montes e Alto Douro, Bragança, Bragança, no date, 1 ex. ( RNC); Beira Litoral, Aveiro, Eirol, Near Soccer Field 20 Jul 1967, 1 ex. (RNFC); Coimbra, Coimbra, Choupal, 9 Nov 1966, 1 ex. (RNFC), Pinhal de Marrocos 7 Apr 1970, 1 ex. (RNFC); Leiria, Ansião, Avelar, 10 Jul 19691 ex. (RNFC); Avelar (Várzea de Cima), 20 Apr 1971, 1 ex. (RNFC).

\section{Ontholestes tessellatus (Geoffroy, 1785)}

This is the first record of this species from Portugal.

New data. Portugal: Trás-os-Montes e Alto Douro, Bragança, Serra da Corôa,Vinhais, Moimenta 15 July 1968, 4 exx. (RNFC); Beira Alta, Viseu, Lamego, Avões 10 July 1963, 4 exx. (RNFC).

Genus Platydracus Thomson, 1858

Type species: Staphylinus stercorarius Olivier, 1795

Members of this genus have most of the pronotum punctate with an inpunctate middle line, anterior angles rounded and greater than 90 degrees. Large lateral setae of head not restricted to the anterior half of the temple. Genus of Neotropical, Nearctic, Palearctic, Ethiopian and Oriental distribution. 


\section{Platydracus flavopunctatus (Latreille, 1804)}

Seabra (1943) stated that this species was first recorded from Portugal by Neves (1941). Ferreira (1962) mentioned this species as Staphylinus flavopunctatus. Gamarra \& Outerelo (2008) recorded the species from Trás-os-Montes e Alto Douro.

New data. Portugal: Trás-os-Montes e Alto Douro, Vila Real, Chaves, Vidago, no date, 1 ex. (RNC).

\section{Platydracus fulvipes (Scopoli, 1763)}

Mentioned from Monchique and Arrifana by Oliveira (1893). Ferreira (1962) mentioned this species as Staphylinus fulvipes. Gamarra \& Outerelo, (2008) indicate it from Beira Litoral and Algarve.

New data. Portugal: Douro Litoral, Porto, Paços de Ferreira, Penamaior, no date, 1 ex. (RNC); Trás-os-Montes e Alto Douro, Vila Real, Vila Pouca de Aguiar, Pedras Salgadas, no date, 1 ex. (RNC).

\section{Platydracus stercorarius (Olivier, 1795)}

Ferreira (1962) recorded it from Portugal. Gamarra \& Outerelo ( 2008) reported this taxon from Trás-os-Montes e Alto Douro and Beira Alta.

New data. Portugal: Trás-os-Montes e Alto Douro, Bragança, Serra de Nogueira, Nogueira, 12 May 1961, 1 ex. (RNFC); Beira Alta, Viseu, Santos Evos, 26 May 1960, 1 ex. (RNFC); Beira Litoral, Coimbra, Coimbra, Santo António dos Olivais, 9 Sep 1971, 1 ex. (RNFC).

\section{Genus Staphylinus Linnaeus, 1758}

Type species: Staphylinus erythropterus Linnaeus, 1758

Staphylinus species have anterior angles of pronotum rounded, head with patches of golden setae and a long lateral seta restricted to the anterior half of the temple. Elytra red, distinctly shorter than pronotum with last segment of labial palpus elongate with the apical margin truncate or pointed. Distribution Neotropical, Nearctic, Palearctic, Ethiopian and Oriental Region.

\section{Staphylinus caesareus Cederhjelm, 1798}

Oliveira (1893) recorded this species from Portugal from Serra de Montesinho, Chaves, Serra da Estrêla and Arrifana. Ferreira (1962) recorded it from Portugal. Gamarra \& Outerelo (2008) mentioned it from Trás-os-Montes e Alto Douro, Beira Alta and Beira Litoral.

New data. Portugal: Beira Litoral, Coimbra, Coimbra, Choupal, 4 Jun 1969, 1 ex. (RNFC), Portela, 11 Oct 1970, 2 ex. (RNFC); Beira Baixa, Castelo Branco, Covilhã, Serra da Estrêla, Penhas da Saúde, 13 Oct 1969, 1 ex. (RNFC).

\section{Staphylinus dimidiaticornis Gemmiger, 1851}

Reported from the North of Portugal by Gamarra \& Outerelo (2008).

Genus Tasgius Stephans, 1829

\section{Type species: Staphylinus pedator Gravenhorst, 1802}

This genus is characterized by pronotum punctate, often with an impunctate mid line with anterior angles rounded with body and femora completely black and with elytra longer than pronotum. Last segment of labial palpus robust with apical margin at a distinct angle. 
In Portugal the species are grouped into two subgenera: Rayacheila Motschulsky, 1845 and Tagius sensu strictus. Distribution Nearctic, Palearctic and Oriental Region.

Subgenus Rayacheila Motschoulsky, 1845

Type species: Tasgius inderiensis Motschoulsky, 1845

\section{Tasgius (Rayacheila) globulifer (Geoffroy, 1785)}

Seabra (1943) cited this species as first recorded from Portugal by Oliveira (1893). Ferreira (1962) recorded it from Portugal. Gamarra \& Outerelo (2008) listed it from Douro Litoral, Beira Litoral, Estremadura and Baixo Alentejo.

\section{Tasgius (Rayacheila) lusitanicus (Muller, 1943)}

Gamarra \& Outerelo (2008) cited this species from Beira Litoral and Estremadura.

\section{Tasgius (Rayachelia) morsitans (Rossi, 1790)}

Seabra (1942) mentioned this species from Portugal from Serra do Gerês as recorded by Oliveira. Cited from Portugal by Gamarra \& Outerelo (2008) from Minho.

\section{Tasgius (Rayachelia) winkleri (Bernhauer, 1906)}

Oliveira (1893) cited this species from Bussaco, Bragança, Espinho and Douro. Gamarra \& Outertelo (2008) listed this species from Trás-os-Montes e Alto Douro, Douro Litoral and Beira Litoral.

Subgenus Tasgius Stephans, 1829

\section{Tasgius (Tasgius) ater (Gravenhorst, 1802)}

Oliveira (1893) cited it from Azambuja and Seabra (1943) as first noted by Heyden in 1870. Ferreira (1962) mentioned this species from Portugal. Gamarra \& Outerelo (2008) recorded this species from Beira Litoral and Alto Alentejo.

\section{Tasgius (Tasgius) pedator (Gravenhorst, 1802)}

Quedenfeldt (1884) mentioned this species from Lisbon. Oliveira (1893) recorded this species from Bragança. Ferreira (1962) recorded it from Portugal. Gamarra \& Outerelo (2008) from Trás-os-Montes e Alto Douro and Estremadura.

\section{ACKNOWLEDGEMENTS}

Special thanks to my son José E. Ferreira for constructive criticism on an early draft of the manuscript and Dr. Jane O'Donnell (University of Connecticut, Storrs) and Dr. William L. Krinsky (Yale University, Peabody Museum of Natural History), for reading and providing comments on the final draft of this manuscript, and to the Editorial Board of Fragmenta Faunistica, specially Dr. Piotr Wegrzynowicz, for inviting me to collaborate in this Journal to commemorate the long life and achievements of a friend and great entomologist Prof. Andrzej Szujecki.

\section{REFERENCES}

COIfFAit H. 1964. Note sur les Ocypous (sensu lato) avec description de forms nouvelles. Bulletin de la Société d'Histoire Naturelle de Toulouse. 99 (1-2): 81-106.

DE LA Fuente J. M. 1922. Catálogo sistemático-geográfico de los Coleópteros observados en la Península Ibérica, Pirineos propriamenta dichos y Baleares. Boletin de la Socieded Entomológica de España 5 (8-9): 126-141. 
FERREIRA R. N. 1962. Contribuições para um catálogo da família Staphylinidae da fauna portuguesa. I - Estado actual do conhecimento dos Estafilinídeos portugueses. Memórias e Estudos do Museu Zoológico da Universidade de Coimbra 280: 1-21.

Gamarra P. \& Outerelo R. 2008. Catálogo Iberobalear de los Staphylinidae (Coleoptera: Staphylinidae). Boletin Sociedad Entomológica Aragonesa 42: 197-251, New Revision, 30 December, 2010.

HEYDEN L. von 1870. Entomologische Reise nach dem sudlichen Spanien, der Sierra Guadarrama und Sierra Morena, Portugal und den Cantabrischen gebirgen, Berlin.

NeVEs R. 1941. Notas Entomológicas. Memórias e Estudos do Museu Zoológico da Universidade de Coimbra 148: 1-5.

OLIVEIRA [M.] P. DE 1893. Catalógue des Insectes du Portugal, Coimbra, 393 pp.

QuEDENFELDT M. 1884. Beiträge zur Kenntniss der Staphylinen - Fauna von Süd-Spanien, Portugal und Marokko. Berliner Entomologische Zeitschrift 28 (2): 351-379.

SeABrA A. F. 1942. Aditamento ao Catálogo dos Coleópteros de Portugal do Dr. Manuel Paulino de Oliveira. Memórias e Estudos do Museu Zoológico da Universidade de Coimbra. 136: 1-33.

SeABrA A. F. 1943. Contribuições para o inventário da Fauna Lusitânica, Insecta, Coleóptera. Memórias e Estudos do Museu Zoológico da Universidade de Coimbra 142: I-XX , 1-152.

SmetanA A. 1959. The Palearctic, Oriental and Nearctic species of the genus Ontholestes GGLB (Col., Staphylinidae). Acta Entomologica Musei Nationalis Pragae 33: 393-412.

SMETANA A. \& DAVIS A. 2000. Reclassification of the north temperate taxa associated with Staphylinus sensu lato, including comments on relevant subtribes of Staphylinini (Coleoptera: Staphylinidae). American Museum Novitates 3287, 88 pp., 159 figures.

\section{STRESZCZENIE}

[Nowe stwierdzenia $i$ dane o rozmieszczeniu gatunków $z$ plemienia Staphylinina (Coleoptera: Staphylinidae: Staphylinini: Staphylinina) z Portugalii]

Artykuł stanowi zaktualizowany przegląd występowania w Portugalii gatunków z plemienia Staphylinina w podrodzinie Staphylininae. W rejestrze uwzględniono 156 okazów reprezentujących 28 gatunków z 8 rodzajów. Znalazły się tu dwa gatunki nowe dla portugalskiej fauny. Dla 25 gatunków i podgatunków podano nowe lokalizacje, poszerzające ich zasięg geograficzny. Niektóre gatunki znane wcześniej z Portugalii nie były ostatnio wykazywane, ale wraz ze wzrostem zbiorów faunistycznych mają jeszcze szanse być znalezione. 